\title{
Análisis en la condición física y el rendimiento académico en niñas adolescentes
}

Mireia Adelantado Renau al117362@uji.es

Diego Moliner Urdiales dmoliner@uji.es 
Objetivos: analizar la relación entre el nivel diario de actividad física y el rendimiento académico y la relación entre el nivel de condición física y el rendimiento académico en un grupo de alumnas de 2. curso de Educación Secundaria Obligatoria.

Metodología: participaron un total de 15 niñas con una edad media de 13,4 $\pm 0,5$ años. 7 de ellas fueron clasificadas como deportistas y las 8 restantes, como sedentarias $(4,6$ vs. 0 sesiones de actividad física a la semana respectivamente). La condición física se evaluó empleando la Batería ALPHA-Fitness; el nivel de desarrollo puberal, mediante la escala autoreportada de Tanner y Whitehouse; la actividad física, a través del cuestionario PAQ-A, y el rendimiento académico mediante las calificaciones finales.

Resultados: las niñas deportistas empleaban un menor número de horas al estudio y la realización de tareas escolares respecto al grupo de no deportistas $(5,3 \pm 2,6$ vs. $8,5 \pm 2,0 ; \mathrm{P}=0,017)$. Las niñas deportistas presentaron niveles de condición física (fuerza de piernas, velocidadagilidad y resistencia) estadísticamente superiores a los presentados por las niñas no deportistas; el rendimiento académico fue similar en ambos grupos ( 6,5 puntos en una escala de 0 a 10).

Conclusiones: la práctica regular de actividad física está asociada a una mejor condición física relacionada con la salud y no parece afectar de forma negativa al rendimiento académico durante la adolescencia.

Palabras clave: actividad física, condición física, rendimiento académico, adolescencia, fuerza, resistencia, sueño, composición corporal.

\section{Introducción}

La adolescencia constituye un periodo de rápido crecimiento en el que se producen múltiples cambios tanto físicos como psicológicos (Shin y Nam, 2015). Durante este periodo crítico del desarrollo humano se adquieren toda una serie de hábitos que pueden influir de forma positiva o negativa durante la etapa adulta (ej. alimentación, práctica de actividad física, consumo de alcohol y tabaco, etc.). Diversos estudios sugieren que los adolescentes gastan un elevado porcentaje de su tiempo libre en actividades sedentarias (p. ej. videojuegos, televisión, ordenador, etc.), duermen menos horas de las recomendadas, mantienen dietas poco equilibradas y se inician en el consumo de alcohol y tabaco a edades tempranas (Moreno y col., 2014). La promoción de hábitos saludables en estas edades ( $p$. ej. fomento de la actividad física, mejora de la condición física, cumplimiento de las horas mínimas de sueño, etc.) constituye una estrategia de salud pública eficaz que reporta múltiples beneficios, no 
sólo a nivel físico sino también psicológico y cognitivo (Paradells y col., 2015).

La actividad física se define como cualquier movimiento corporal producido por la acción de la musculatura que incrementa el gasto de energía por encima de los valores basales (Ortega y col., 2008 b). Entre los principales factores que definen la actividad física se incluyen la intensidad, la frecuencia, la duración y el tipo. La práctica regular de actividad física conlleva múltiples efectos positivos sobre la salud en niños y adolescentes (Penedo y Dahn, 2005), y ayudan al óptimo desarrollo del sistema músculo-esquelético y cardiovascular, mejoran la salud mental y minimizan el riesgo de padecer patologías durante la etapa adulta. Sin embargo, la adolescencia es un periodo en el que se reduce significativamente la actividad física, especialmente en chicas (DjordjevicNikic y col., 2013), lo que incrementa el riesgo de padecer obesidad (Galson, 2008) y alteraciones metabólicas graves como diabetes, hipertensión e hiperlipidemia (Vaynman y Gomez-Pinilla, 2006), a la vez que afecta negativamente a la salud cognitiva y a las funciones cerebrales (Chaddock y col., 2011).

Las actuales recomendaciones de actividad física para la salud establecen que un adolescente activo es aquel que realiza al menos 60 minutos diarios de actividad física de intensidad moderada o intensa (Ortega y col., 2008 a). Además de los múltiples beneficios para la salud derivados de esta práctica regular de actividad física durante la adolescencia, nuevos estudios parecen sugerir que este hábito podría estar asociado a una mejora de la capacidad cognitiva y del rendimiento académico (Hillman y col., 2008), aunque todavía son necesarias nuevas investigaciones que corroboren estos resultados.

La condición física relacionada con la salud se define como la capacidad para realizar las actividades de la vida diaria con vigor, y se diferencian 4 componentes: la resistencia cardiorrespiratoria, la fuerza muscular, la capacidad motora y la composición corporal (Ruiz y col., 2009). Numerosos estudios ponen de manifiesto que una buena condición física se asocia con un menor riesgo de enfermedades crónicas y muerte prematura en la etapa adulta y se considera desde las primeras edades como un potente marcador de salud (Ortega y col., 2008 b). Incluso recientes investigaciones indican que una buena condición física en la adolescencia podría estar asociada a una mejor capacidad cognitiva y un mayor rendimiento académico (Khan y Hillman, 2014), aunque son necesarios nuevos estudios que permitan validar estos resultados.

El rendimiento académico hace referencia al nivel de conocimientos y habilidades que un alumno demuestra a través de un sistema de evaluación. Habitualmente, las investigaciones recurren a las calificaciones obtenidas en las asignaturas instrumentales (Lengua y Matemáticas) como principales indicadores del rendimiento académico. El informe Programme for International Student Assessment (PISA) pone de manifiesto el bajo nivel del rendimiento académico de los adolescentes españoles en comparación con el resto de países de la 
Organización para la Cooperación y el Desarrollo Económico (OCDE). Según los datos obtenidos en 2012, los adolescentes españoles ocupan una posición por debajo de la media de la OCDE.

Diversos estudios asocian el rendimiento académico y la capacidad cognitiva con el nivel de actividad física y condición física en adolescentes. Taras (2005) pone de manifiesto la existencia de una relación positiva entre la práctica regular de actividad física y el rendimiento académico en adolescentes. Estudios recientes sugieren que determinados componentes de la condición física (fuerza, velocidad, flexibilidad, agilidad, etc.) podrían mejorar funciones del cerebro y el rendimiento académico (Esteban-Cornejo y col., 2014).

A pesar del creciente número de estudios científicos centrados en el análisis de las relaciones que se establecen entre la actividad física, la condición física y el rendimiento académico, los resultados obtenidos no son concluyentes, de manera que son necesarios nuevos estudios que permitan ampliar el nivel actual de conocimiento científico.

\section{Objetivos}

Los objetivos del presente estudio son analizar la relación entre el nivel diario de actividad física y el rendimiento académico y analizar la relación entre el nivel de condición física y el rendimiento académico en un grupo de alumnas de 2 o curso de Educación Secundaria Obligatoria con diferentes niveles de actividad física.

\section{Material y método}

\section{Participantes y procedimiento}

Durante el curso académico 2014-2015 un total de 20 niñas adolescentes de institutos públicos de Castellón fueron invitadas a participar en el estudio. Finalmente, 15 niñas aceptaron participar y fueron incluidas tras comprobar que cumplían todos los criterios de inclusión establecidos: nacidas en el año 2001, cursando 2. de Educación Secundaria Obligatoria en un centro educativo de carácter público, sin limitaciones o patologías crónicas de tipo físico ni cognitivo y practicantes de actividades deportivas competitivas con al menos 3 sesiones de entrenamiento semanal o no practicantes de ningún tipo de actividad deportiva.

Las 15 niñas seleccionadas presentaban una edad media de 13,4 \pm 0,5 años, y se dividieron en dos grupos según su nivel de actividad física. Un total de 7 niñas fueron clasificadas como deportistas, mientras que las 8 restantes fueron clasificadas como no deportistas.

Todas las adolescentes participaron de forma voluntaria en el presente estudio $y$ sus padres $o$ tutores legales firmaron un 
consentimiento informado. El estudio fue aprobado por la Comisión Deontológica de la Universidad Jaume I.

\section{Composición corporal y condición física}

Las medidas para la determinación de la composición corporal se realizaron en una habitación habilitada para ello y convenientemente climatizada para obtener una óptima sensación de confort. Todas las mediciones se realizaron de forma individual bajo la presencia de un investigador cualificado y un ayudante.

El peso fue medido en ropa interior y sin zapatos, mediante una balanza (SECA 861) con una precisión de 0,1 kg. Se realizaron dos medidas y se empleó la media de ambas para los análisis. La altura se determinó mediante un tallímetro (SECA 225) con una precisión de $0,1 \mathrm{~cm}$. Cada niña era colocada con los talones juntos y con los brazos a lo largo del cuerpo. Los talones, glúteos y parte superior de la espalda estaban en contacto con el tallímetro. La cabeza se orientaba de tal manera que quedaban en un mismo plano horizontal la protuberancia superior del tragus del oído y el borde inferior de la órbita del ojo (plano de Frankfort). La medición se realizaba en el momento en el que cada niña inspiraba profundamente y mantenía la respiración. Se tomó como referencia el punto más alto. Se realizaron dos medidas y se empleó la media de ambas para los análisis.

El IMC (índice de masa corporal) fue calculado como el cociente del peso de cada niña en kilogramos entre el cuadrado de su altura en metros.

La condición física se evaluó mediante la aplicación de la Batería ALPHA-Fitness (Ruiz y col., 2011). La Batería ALPHA-Fitness consta de una serie de tests de campo que permiten la evaluación de la condición física relacionada con la salud en niños y adolescentes.

La capacidad aeróbica fue medida mediante la realización del test de ida y vuelta de $20 \mathrm{~m}$ : cada niña se desplazaba entre dos líneas situadas a 20 metros de distancia, debiendo hacer un cambio de sentido al llegar a cada una de ellas y coincidiendo con el ritmo indicado por una señal sonora que iba acelerándose progresivamente. La velocidad inicial de la señal era de $8,5 \mathrm{~km} / \mathrm{h}$, y se incrementaba en $0,5 \mathrm{~km} / \mathrm{h}$. La prueba terminaba cuando la niña no era capaz de llegar por segunda vez consecutiva a una de las líneas con la señal de audio o cuando se detenía debido a la fatiga. Se registraba el número total de rectas completadas.

La fuerza de prensión manual fue evaluada mediante un dinamómetro manual (TKK 5401; Takei, Tokyo, Japan) con una precisión de $0,5 \mathrm{~kg}$. Cada niña apretaba el dinamómetro manual gradualmente y de forma continua durante al menos 2 segundos. El dinamómetro se ajustaba al tamaño de la mano. El codo quedaba extendido evitando que el dinamómetro contactara con cualquier parte del cuerpo. El test se realizaba en dos ocasiones con cada una de las manos. Se escogía el valor más alto de las dos medidas realizadas con cada mano y se realizaba la media de estos dos valores, obteniéndose así la fuerza media de prensión manual. 
La fuerza explosiva del tren inferior se midió utilizando el test de salto en longitud. Cada niña colocaba sus pies juntos tras la línea de salto y con una separación igual a la anchura de sus hombros, doblaba las rodillas a la vez que colocaba los brazos delante del cuerpo y paralelos al suelo. Se balanceaba y realizaba el salto lo más lejos posible tomando contacto con el suelo con los dos pies simultáneamente. El test se realizaba en dos ocasiones y se registraba el resultado más alto de los dos intentos en centímetros.

La capacidad de velocidad/agilidad se determinó mediante la prueba de velocidad agilidad 4×10 metros. En el suelo se dibujaban dos líneas paralelas a 10 metros de distancia. Cuando se indicaba la salida, la niña corría lo más rápido posible de una línea a la otra hasta completar la distancia de 10 metros en 4 ocasiones. El test se realizaba en dos ocasiones y el mejor resultado era anotado. El resultado se registraba en segundos con un decimal.

\section{Desarrollo puberal}

El desarrollo puberal se determinaba empleando la escala autoreportada de estadios madurativos de Tanner y Whitehouse (Tanner y Whitehouse, 1976). A cada participante se le mostraba la escala de Tanner con los diversos estadios, de forma que únicamente tenía que indicar su nivel de desarrollo respecto a los senos y el vello púbico.

\section{Actividad física}

La actividad física se evaluó empleando el cuestionario PAQ-A (Physical Activity Questionnaire for Adolescents) completado en un tiempo máximo de 15 minutos. Este cuestionario consiste en 9 preguntas y fue diseñado para valorar la actividad física en los últimos 7 días empleando una escala de Likert de 5 puntos. El resultado final obtenido es una puntuación que permite establecer el nivel de actividad física de cada niña (Martínez-Gómez y col., 2009).

\section{Rendimiento académico}

El rendimiento académico se estableció a partir de las calificaciones finales obtenidas por cada una de las niñas en el curso anterior (1. de ESO). Cada niña aportó el certificado original de notas proporcionado por su centro educativo. Para este análisis exclusivamente se utilizaron las calificaciones finales correspondientes a las asignaturas instrumentales: Castellano: Lengua y Literatura, Valencià: Llengua i Literatura, Lengua Extranjera: Inglés y Matemáticas. Se calculó la media aritmética de todas las asignaturas instrumentales para tener una valoración global del rendimiento académico. 


\section{Cuestionarios}

Las adolescentes completaron un breve cuestionario en el que se les preguntó información sobre diversas variables que podrían tener influencia sobre su rendimiento académico.

- Para conocer las horas de sueño se les preguntó cuántas horas de sueño real tenían cada noche desde que se iban a la cama hasta que se levantaban por la mañana.

- Para determinar el tiempo dedicado al estudio y a la realización de tareas escolares se les preguntó cuántas horas dedicaban un día entre semana y cuántas horas dedicaban en un día de fin de semana a estudiar y realizar tareas escolares. Para calcular los valores semanales de horas de estudio y tareas escolares se multiplicaron las horas que dedicaban a ello en un día entre semana por cinco y las horas que dedicaban al estudio en un día de fin de semana por dos. Los resultados obtenidos se sumaron y se obtuvieron las horas semanales de estudio.

- El máximo nivel de estudio alcanzado por sus padres fue reportado a través de la siguiente escala: sin estudios, educación primaria, educación secundaria, estudios de bachillerato, estudios universitarios.

\section{Análisis estadísticos}

Las características de la muestra analizada en el estudio se presentan como medias y desviaciones estándar. Las diferencias entre el grupo de niñas deportistas y no deportistas se calcularon utilizando el test de la $\mathrm{t}$ de Student.

Todos los análisis fueron llevados a cabo utilizando la versión 23.0 del paquete estadístico SPSS, considerando $\mathrm{P}<0,05$ como estadísticamente significativa.

\section{Resultados}

La muestra de participantes estaba formada por 7 niñas adolescentes deportistas que realizaban al menos 4 sesiones semanales de actividad deportiva y 8 niñas no deportistas que no realizaban ningún tipo de actividad física regular $(P<0,000)$.

Tabla 1. Características generales de las participantes del estudio

\begin{tabular}{lccc}
\hline & $\begin{array}{c}\text { DEPORTISTAS } \\
\mathbf{n = 7}\end{array}$ & $\begin{array}{c}\text { NO DEPORTISTAS } \\
\mathbf{n = 8}\end{array}$ & Valores $\mathbf{P}$ \\
\hline Edad (años) & $13,4 \pm 0,5$ & $13,1 \pm 0,4$ & 0,230 \\
\hline Tanner (estadio) & $3,4 \pm 0,5$ & $3,4 \pm 0,5$ & 0,847 \\
\hline Peso $(\mathrm{kg})$ & $54,5 \pm 5,4$ & $51,0 \pm 5,0$ & 0,215 \\
\hline Talla (cm) & $165,5 \pm 6,7$ & $159,6 \pm 7,1$ & 0,125 \\
\hline
\end{tabular}




\begin{tabular}{|c|c|c|c|}
\hline $\mathrm{IMC}\left(\mathrm{kg} / \mathrm{m}^{2}\right)$ & $19,9 \pm 1,7$ & $20,1 \pm 2,0$ & 0,892 \\
\hline Horas estudio (semana) & $5,3 \pm 2,6$ & $8,5 \pm 2,0$ & 0,017 \\
\hline Sueño (h/día) & $7,7 \pm 1,0$ & $7,1 \pm 0,8$ & 0,223 \\
\hline AF (días/semana) & $4,6 \pm 0,5$ & $0,0 \pm 0,0$ & $<0,000$ \\
\hline \multicolumn{4}{|c|}{ IMC indica índice de masa corporal } \\
\hline \multicolumn{4}{|c|}{ Valores P calculados mediante el test de la t de Student } \\
\hline
\end{tabular}

No se observaron diferencias estadísticamente significativas entre los grupos respecto a los valores referidos a los parámetros físicos. El índice de masa corporal para ambos grupos era de $20 \mathrm{~kg} / \mathrm{m}^{2}$ y todas las niñas manifestaron dormir una media de 7 horas diarias. Se observaron diferencias estadísticamente significativas respecto a la variable horas de estudio, donde las niñas no deportistas manifestaron dedicar más de 8 horas semanales frente a las 5 que indicaron la niñas deportistas $(P=0,017)$.

Tabla 2. Nivel de condición física de las participantes del estudio

\begin{tabular}{lccc}
\hline & $\begin{array}{c}\text { DEPORTISTAS } \\
\mathbf{n = 7}\end{array}$ & $\begin{array}{c}\text { NO DEPORTISTAS } \\
\mathbf{n = 8}\end{array}$ & Valores P \\
\hline Fuerza mano derecha $(\mathrm{kg})$ & $29,0 \pm 2,1$ & $26,2 \pm 4,0$ & 0,116 \\
\hline Fuerza mano izquierda $(\mathrm{kg})$ & $29,8 \pm 4,5$ & $24,6 \pm 5,0$ & 0,053 \\
\hline Fuerza media manos $(\mathrm{kg})$ & $29,4 \pm 2,9$ & $25,4 \pm 4,4$ & 0,059 \\
\hline Salto $(\mathrm{cm})$ & $192,9 \pm 17,3$ & $141,9 \pm 12,5$ & $<0,000$ \\
\hline Velocidad (seg.) & $12,4 \pm 0,5$ & $13,8 \pm 0,7$ & 0,001 \\
\hline Resistencia (rectas) & $82,9 \pm 16,9$ & $38,9 \pm 11,6$ & $<0,000$ \\
\hline
\end{tabular}

Los niveles de condición física presentaron diferencias estadísticamente significativas entre los dos grupos. Excepto para las variables de fuerza de prensión manual, las niñas deportistas presentaron unos niveles de fuerza de piernas, velocidad-agilidad y resistencia estadísticamente superiores a los presentados por el grupo de niñas no deportistas.

Tabla 3. Rendimiento académico de las participantes del estudio

\begin{tabular}{lccc}
\hline & DEPORTISTAS & NO DEPORTISTAS & \multirow{2}{*}{ Valores $\mathbf{P}$} \\
& $\mathbf{n = 7}$ & $\mathbf{n = 8}$ & \\
\hline Castellano: Lengua y Literatura & $6,9 \pm 1,6$ & $6,1 \pm 1,1$ & 0,314 \\
\hline Valencià: Llengua i Literatura & $6,6 \pm 1,1$ & $7,0 \pm 1,5$ & 0,550 \\
\hline Lengua Extranjera: Inglés & $6,3 \pm 1,4$ & $6,8 \pm 2,0$ & 0,613 \\
\hline Matemáticas & $6,9 \pm 1,9$ & $6,1 \pm 0,8$ & 0,366 \\
\hline Media & $6,6 \pm 1,4$ & $6,5 \pm 1,2$ & 0,836 \\
\hline $\begin{array}{l}\text { Media indica la media aritmética de las calificaciones obtenidas en castellano, valenciano, } \\
\text { inglés y matemáticas } \\
\text { Valores P calculados mediante el test de la t de Student. }\end{array}$ & & \\
\hline
\end{tabular}

En relación con el rendimiento académico ambos grupos presentaron valores muy similares en todas las variables analizadas, sin identificarse diferencias estadísticamente significativas. La nota media de las materias 
instrumentales (Castellano: Lengua y Literatura, Valencià: Llengua i Literatura, Lengua Extranjera: Inglés y Matemáticas) fue de un $\approx 6,5$ para ambos grupos.

\section{Discusión y conclusiones}

En el presente estudio los datos obtenidos parecen indicar que la práctica regular de actividad física no influye negativamente sobre el rendimiento académico y que además conlleva una mejora notable de la condición física relacionada con la salud.

Nuestros resultados coinciden con los obtenidos por Daley y Ryan (2000), donde en un estudio con 232 adolescentes no encontraron correlación alguna entre la práctica regular de actividad física y el rendimiento académico. Estudios similares como los llevados a cabo por Bass y col., y Haapala (2013) sugieren la existencia de una relación positiva entre el rendimiento académico y la práctica de actividad física.

Las niñas deportistas evaluadas en nuestro estudio presentaban parámetros físicos (IMC, horas de sueño, peso, talla y Tanner) muy similares a las no deportistas, lo que pone de manifiesto que se trataba de dos grupos muy homogéneos. Ambos grupos presentaban diferencias significativas respecto al nivel de actividad física y las horas de estudio semanales. De hecho, las niñas no deportistas dedicaban al estudio y tareas escolares una media de $8,5 \pm 2,0$ horas a la semana, mientras que las deportistas empleaban de media 5,3 $\pm 2,6$ horas semanales. Todas ellas tenían la misma jornada escolar y afirmaban dormir $\approx 7 \mathrm{~h}$ al día. Por tanto, el hecho de que las deportistas dediquen menos horas al estudio podría deberse a que empleaban parte de su tiempo en entrenar y preparar sus competiciones, mientras que las niñas no deportistas como no realizaban ningún tipo de actividad deportiva tenían más tiempo libre disponible para dedicar al estudio y a la realización de tareas escolares.

Los niveles de condición física presentaron diferencias estadísticas entre el grupo de deportistas y no deportistas para todas las variables analizadas excepto para la fuerza de prensión manual. Los resultados obtenidos por el grupo de deportistas fueron muy superiores en las pruebas de velocidad, salto y resistencia. Probablemente esto sea debido a que el grupo de niñas deportistas practicaba atletismo, deporte en el que el uso de las extremidades superiores es muy limitado, mientras que las extremidades inferiores están en continua solicitación y, por tanto, presentan un nivel de desarrollo mayor.

Investigaciones recientes ponen de manifiesto una relación positiva entre la condición física y el estado de salud física y psicológica (Lankhorst y col., 2015). Además, nuevas líneas de investigación parecen apuntar que tanto la mejora de la resistencia cardiorrespiratoria, como de la fuerza muscular están asociadas a un mayor rendimiento académico en adolescentes (Kalantari y Esmaeilzadeh, 2015; Sardinha y col., 2015).

Los resultados de nuestro estudio muestran que la práctica de actividad física no influye de forma negativa sobre el rendimiento 
académico. De hecho, ninguna de las variables de actividad física presentó una relación significativa con las variables del rendimiento académico. A pesar del elevado volumen de horas que invierten las niñas deportistas en su práctica deportiva y al menor tiempo empleado en el estudio y la realización de tareas escolares, su rendimiento académico ( $\approx 6.5)$ fue similar al de las niñas no deportistas. Consideramos que estos resultados podrían deberse en parte a la mejora de la plasticidad cerebral, al incremento de la capacidad de concentración y de la memoria y a la adquisición de habilidades académicas (Kamijo y col., 2012) que se asocian a la práctica regular de actividad física. De hecho, la práctica regular de actividad física presenta múltiples efectos positivos durante la adolescencia, ya que ayuda a controlar el estrés, a gestionar mejor el tiempo y además transmite valores asociados a la cultura del esfuerzo (Hansmann y col., 2007; Norris y col., 1992).

Entre los puntos fuertes de nuestro estudio cabe destacar que se trata de una muestra muy homogénea que presenta niveles muy diferentes de actividad física. Además, para analizar la relación entre la actividad física y el rendimiento académico se han tenido en cuenta la influencia de diversas variables de confusión como las horas de estudio semanales, el nivel de condición física y las horas de sueño diarias. Sin embargo, estos resultados han de ser interpretados con cautela debido al reducido tamaño de la muestra compuesta exclusivamente por niñas, el uso de cuestionarios para evaluar la actividad física y a la falta de control sobre otras variables que pueden influir en el rendimiento académico como la dieta, el nivel socioeconómico familiar o variables sociodemográficas (Torrijos-Niño y col., 2014). Además, el diseño transversal de nuestro estudio no nos permite establecer relaciones causa-efecto.

De acuerdo con el conocimiento científico actual y con los resultados encontrados en nuestro estudio podemos afirmar que la práctica regular de actividad física mejora la salud de las niñas adolescentes sin afectar de forma negativa a su rendimiento académico. Tanto las familias como las instituciones educativas deberían tener en cuenta estas evidencias a la hora de tomar decisiones orientadas a la promoción de la salud y la mejora del rendimiento académico durante la infancia y la adolescencia.

\section{Agradecimientos}

Los autores quieren dar las gracias a todos los participantes en el estudio por su implicación, y a todos los miembros del grupo de investigación LIFE de la Universitat Jaume I por el trabajo realizado durante la toma de datos.

El presente trabajo constituye un estudio piloto enmarcado dentro del proyecto DADOS que ha sido financiado por el Ministerio de Economía y Competitividad a través del Programa Estatal I+D+l Orientada a los Retos de la Sociedad en la convocatoria del año 2013 (Ref. Dep201345515-R) y cuenta con el patrocinio del Grupo Schweppes. 


\section{Bibliografía}

BASS, R. W. y otros (2013): "Physical Fitness and Academic Performance in Middle School Students», Acta Paediatrica, 102(8), p. 832-837.

CHADDOCK, L. y otros (2011): «A Review of the Relation of Aerobic Fitness and Physical Activity to Brain Structure and Function in Children», Journal of the International Neuropsychological Society, 17(6), p. 975-985.

Daley, A. J. y J. Ryan (2000): «Academic Performance and Participation in Physical Activity by Secondary School Adolescents», Perceptual and Motor Skills, 91(2), p. 531-4.

DJORDJEVIC-NIKIC, M. y otros (2013): «Nutritional and Physical Activity Behaviours and Habits in Adolescent Population of Belgrade», Vojnosanitetski Pregled, 70(6), p. 548-554.

Esteban-CoRnejo, I. y otros (2014): «Independent and Combined Influence of the Components of Physical Fitness on Academic Performance in Youth", The Journal of Pediatrics, 165(2), p. 306-312.e2.

GaLSON, S. K. (2008): "Childhood Overweight and Obesity Prevention», Public Health Reports (Washington, D.C. : 1974), 123(3), p. 258-9.

HaApala, E. A. (2013): "Cardiorespiratory Fitness and Motor Skills in Relation to Cognition and Academic Performance in Children - a Review», Journal of Human Kinetics, 36, p. 55-68.

HANSMANN, R. y otros (2007): «Restoration and Stress Relief Through Physical Activities in Forests and Parks», Urban Forestry \& Urban Greening, 6(4), p. 213-225.

HILLMAN, C. H. y otros (2008): «Be Smart, Exercise Your Heart: Exercise Effects on Brain and Cognition", Nature Reviews. Neuroscience, 9(1), p. 58-65.

KalantARI, H.-A., y S. EsmaeILZAdeh (2015): «Association Between Academic Achievement and Physical Status Including Physical Activity, Aerobic and Muscular Fitness Tests in Adolescent Boys», Environmental Health and Preventive Medicine.

KamiJo, K. y otros (2012): "The Relation of Adiposity to Cognitive Control and Scholastic Achievement in Preadolescent Children", Obesity (Silver Spring, Md.), 20(12), p. 2406-11.

Khan, N. y C. H. Hillman (2014): "The Relation of Childhood Physical Activity and Aerobic Fitness to Brain Function and Cognition: a Review», Pediatric Exercise Science, 26(2), p. 138-46.

LANKHORST, K. y otros (2015): "Health in Adapted Youth Sports Study (HAYS): Health Effects of Sports Participation in Children and Adolescents with a Chronic Disease or Physical Disability», SpringerPlus, 4, p. 796. 
Martínez-Gómez, D. y otros (2009): "Reliability and Validity of the PAQ-A Questionnaire to Assess Physical Activity in Spanish Adolescents», Rev Esp Salud Pública, 83, p. 427-439.

MoRenO, L. A. y otros (2014): «Nutrition and Lifestyle in European Adolescents: the HELENA (HealthyLiftestyle in Europe by Nutrition in Adolescence) Study", Advances in Nutrition, 5(Part 2), p. 615A-623AS.

NoRRIS, R. y otros (1992): "The Effects of Physical Activity and Exercise Training on Psychological Stress and Well-being in an Adolescent Population", Journal of Psychosomatic Research, 36(1), p. 55-65.

ORTEGA, F. B. y otros (2008 a): "Los adolescentes físicamente activos presentan una mayor probabilidad de tener una capacidad cardiovascular saludable independientemente del grado de adiposidad». The European Youth Heart Study. Revista Española de Cardiología, 61(2), p. 123-129.

- «Physical Fitness in Childhood and Adolescence: a Powerful Marker of Health», International Journal of Obesity, 32(1), p. 1-11.

Paradells, V. N., y otros (2015): «Effects of an Intervention Program (HHP) on the Promotion of Healthy Habits in Early Adolescence», Nutr Hosp., $32(6)$, p. 2640-2649.

Penedo, F. J. y J. R. Dahn (2005): «Exercise and Well-being: a Review of Mental and Physical Health Benefits Associated with Physical Activity", Current Opinion in Psychiatry, 18(2), p. 189-93.

RuIz, J. R. y otros (2009): "Predictive Validity of Health-related Fitness in Youth: a Systematic Review», British Journal of Sports Medicine, 43(12), p. 909-923.

RUIz, J. R. y otros (2011): "Batería ALPHA-Fitness : test de campo para la evaluación de la condición física relacionada con la salud en niños y adolescentes», Nutrición Hospitalaria, 26(6), 1210-1215.

SARDINHA, L. B. y otros (2015): «Longitudinal Relationship Between Cardiorespiratory Fitness and Academic Achievement», Medicine and Science in Sports and Exercise.

ShIN, A., y C. M. NAM (2015): "Weight Perception and its Association with Socio-demographic and Health-related Factors among Korean Adolescents». BMC Public Health, 15(1), p. 1292.

TANNeR, J. M., y R. H. Whitehouse (1976): «Clinical Longitudinal Standards for Height, Weight, Height Velocity, Weight Velocity, and Stages of Puberty", Archives of Disease in Childhood, 51(3), p. 170-179.

TARAS, H. (2005): "Physical Activity and Student Performance at School», The Journal of School Health, 75(6), p. 214-8.

TORRIJOS-NIÑo, C. y otros (2014): «Physical Fitness, Obesity, and Academic Achievement in Schoolchildren", Journal of Pediatrics, 165, p. 104-109.

Vaynman, S., \& Gomez-Pinilla, F. (2006): «Revenge of the "Sit": How 
Mireia Adelantado Renau y Diego Moliner Urdiales. Análisis en la condición física y el rendimiento académico en niñas adolescentes

Lifestyle Impacts Neuronal and Cognitive Health Through Molecular Systems That Interface Energy Metabolism with Neuronal Plasticity", Journal of Neuroscience Research, 84(4), p. 699-715. 